Communications in Physics, Vol. 24, No.3S1 (2014), pp. 64-70

DOI:10.15625/0868-3166/24/3S1/5221

\title{
INVESTIGATION OF THERMAL ANNEALING CONDITION ON THE OPTICAL AND ELECTRICAL PROPERTIES OF HYBRID SILVER NANOWIRES/REDUCED GRAPHENE OXIDE (AgNWS/rGO) FILMS
}

\author{
HOANG THI THU, HUYNH TRAN MY HOA, PHAM HOAI PHUONG, \\ NGUYEN HUYNH NHU, AND TRAN QUANG TRUNG \\ University of Natural Science, Vietnam National University, Ho Chi Minh City \\ LAM MINH LONG AND NGUYEN NANG DINH \\ University of Engineering and Technology, Vietnam National University, Hanoi \\ E-mail: tqtrung@hcmus.edu.vn \\ Received 04 April 2014 \\ Accepted for publication 24 May 2014
}

\begin{abstract}
Herein we report a electrode in the hybrid structure of the silver nanowires (AgNWs) with reduced graphene oxide ( $r G O)$ deposited on glass or PET substrate. The assembly and contacting in procedures of this hybrid film have been strongly affected by preparation conditions, especially annealing temperature. In this work, we have investigated the effects of thermal annealing on interconnected at nanowire junctions and between AgNWS network and rGO films via their sheet resistance and transmission. The annealing temperature was varied from $120^{\circ} \mathrm{C}$ to $180^{\circ} \mathrm{C}$ with $10^{\circ} \mathrm{C}$ step to find out optimal temperature at which the wires can be joined together. The results show that at $170^{\circ} \mathrm{C}$, the sheet resistance and transmission of the hybrid rGO/Ag NW film are $R_{S}=10.7 \Omega s q^{-1}$ and $T=77 \%$ (at wavelength 550 $n m)$ corresponding to the ratio of direct conductivity to optical conductivity $\sigma_{D C} / \sigma_{O P}=126$ which is the best obtained value. It is expected that the hybrid AgNWS/rGO film can replace ITO film in the near future.
\end{abstract}

Keywords: reduced graphene oxide ( $r G O)$, Hybrid silver nanowires, thermal annealing.

\section{INTRODUCTION}

Conducting metal oxide films such as indium tin oxide (ITO) films and aluminum doped zinc oxide films have been widely used as transparent conductors in a variety of optoelectronic devices including liquid crystal displays, organic light emitting diodes, and thin film solar cells owing to their high optical transparency and high electrical conductivity [1-9]. However, ITO sputtering techniques have several drawbacks such as brittleness and high processing temperature. Furthermore, the scarcity of indium resources makes ITO transparent electrode very expensive recently [4]. Therefore, cheap, flexible, and solution-processable transparent electrodes have been required for next generation of optoelectronic devices such as flexible solar cells and displays [14]. In this work, we attempt to resolve nearly all of the current problems with hybrid AgNWs and reduced graphene oxide by employing spin coating rGO onto pre-existing AgNWs networks at low temperatures. Embedding the AgNWs network in the conductive rGO matrix offers many 
advantages including improved wire to wire junction conductance, smooth surface morphology, excellent mechanical adhesion, flexibility and particularly, AgNWs is protected from oxidation in ambient $[5,6]$.

\section{EXPERIMENT}

The AgNWs material was synthesized by polyol method and GO material was prepared by modified Hummer method at our laboratory. More details were presented in our previous studies [2,3]. The AgNWs dispersion was sprayed onto a $1.2 \mathrm{~cm} \times 1.2 \mathrm{~cm}$ preheated glass (or PET) substrate using an airbrush system with Ar as a carrier gas. An airbrush with a nozzle diameter of $0.2 \mathrm{~mm}$ was set in single action mode to fix a ratio of AgNWs dispersion to Ar. The inlet pressure of Ar was 2 bar. The spraying rate was $0.2 \mathrm{ml} / \mathrm{min}$. The distance between the tip of the nozzle and the substrate was $12 \mathrm{~cm}$. The AgNWs dispersion was atomized into small droplets which were carried by Argon gas toward the preheated substrate. Then, GO was spin coated on AgNWs network directly and was reduced into a rGO by hydrazine in 30 minutes at room temperature.

It is convenient to compare optical and electrical properties of the hybrid thin films, 7 samples are fabricated with the same conditions [2], only changing the annealing temperature.

Finally, the rGO/AgNWs hybrid films were annealed in a tube furnace from $120^{\circ} \mathrm{C}$ to $180^{\circ} \mathrm{C}$ for 30 min to maximize adhesion between the AgNWs and $\mathrm{rGO}$ and to remove the remaining PVP layers on the surface of the AgNWs. The highest annealed temperature in procedure of experiment was selected $180^{\circ} \mathrm{C}$ (the glass transition point of the PET substrate).

Optical transparency at the wavelength of $550 \mathrm{~nm}$ was measured using a UVVis spectrophotometer (JASCO Corp., Tokyo, Japan) with a glassor PET substrate as a reference. The morphology of the samples were observed using a scanning electron microscope (SEM) (JSM-6700F, JEOLLtd., Tokyo, Japan) and Atomic force microscope (AFM) (University of Ulsan, Daehak-ro 102, Nam-gu, Ulsan 680-749, South Korea).The sheet resistances ofthe hybrid films (before and after bending test) were measured using the four-probe method.

\section{RESULTS AND DISCUSSION}

rGO is usually prepared by using chemical method that is the most suitable for the largescale production of single rGO at low cost but the main drawbacks of these method are the use of strong oxidizing agents make rGO sheets were small pieces (as show in Fig. 1a) leading to extremely decrease electrical conductivity of rGO films. For improving their conductive properties, we have attempted to deposit more and more layers of rGO on the substrates (as shown in Fig. 1b). This way is efficiency to decrease the resistance of the electrode but also decrease its transmittance. The low transparent of thick rGO films were not good for TCE (transparent conducting electrode) applications. Therefore, in this work we demonstrate the assembly of rGO with AgNWs in which AgNWs play a role bridge between rGO islands as shown in Fig. 1c. The benefit of using this hybrid films not only to connect the rGO islands to improve conductivity of rGO films but also to maintain the high transmittance of the thin rGO layer [5]. In this proceeding, the improvement of good contact among AgNWs network is extremely important and the annealing temperature is the key for solving this problem. The heat treatment is not only removing the remained PVP on the surface of the AgNWs but also fuses the AgNWs together such that tight connections leading to 
high conductivity. Hence, these films significantly decrease the resistance down to $10.7 \Omega / \square$ and exhibit optical transmittance (77\%). This result promise hybrid AgNWs/rGO can be replaced ITO in display application in near future.
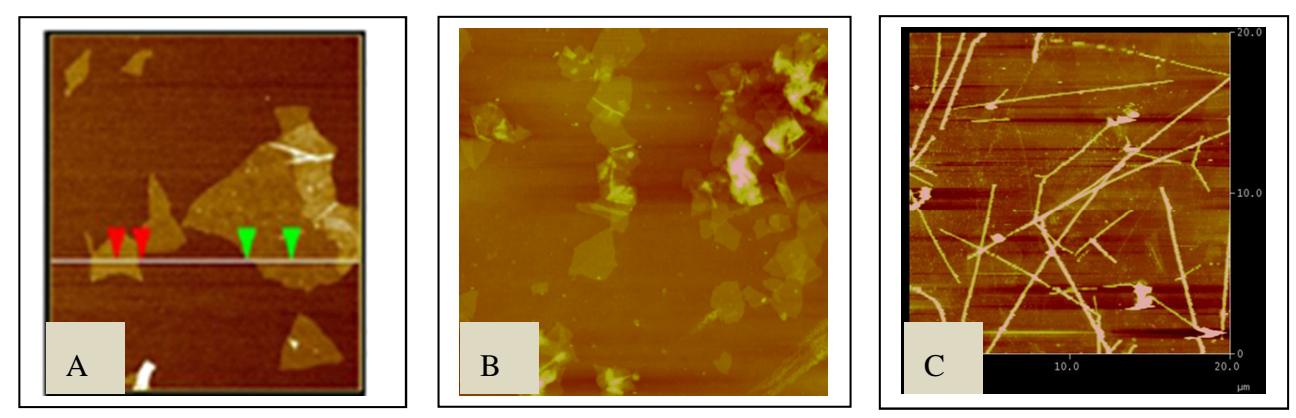

Fig. 1. AFM image of rGO islands (A), percolation of rGO sheets (B), AgNWs are play bridging between rGO islands (scale $20 \mu \mathrm{m}$ ).

Fig. 2(a) shows SEM images of AgNWs synthesized by a polyol process before annealing,indicating that the AgNWs have an average diameter of $50 \mathrm{~nm}$ and length upper $10 \mu \mathrm{m} \mathrm{[2].}$ The insert in Fig. 2(a) demonstrates the percolation of discrete AgNWs in which the contact point between two AgNWs seem as physical contact. In contrast, Fig. 2(b) shown SEM images of Ag$\mathrm{NWs}$ films after $170^{\circ} \mathrm{C}$ annealing in 30 min that exhibited the melting and fusion of the AgNWs at contact points. As the results, the sheet resistant of network decreased significantly. Follow Young Soo Yun [8], melting and fusion of the AgNWs after thermal annealing significantlyalso increased their contacts to the GO layers, resulting in mechanically stable and flexible TCE as shown in Fig. 3
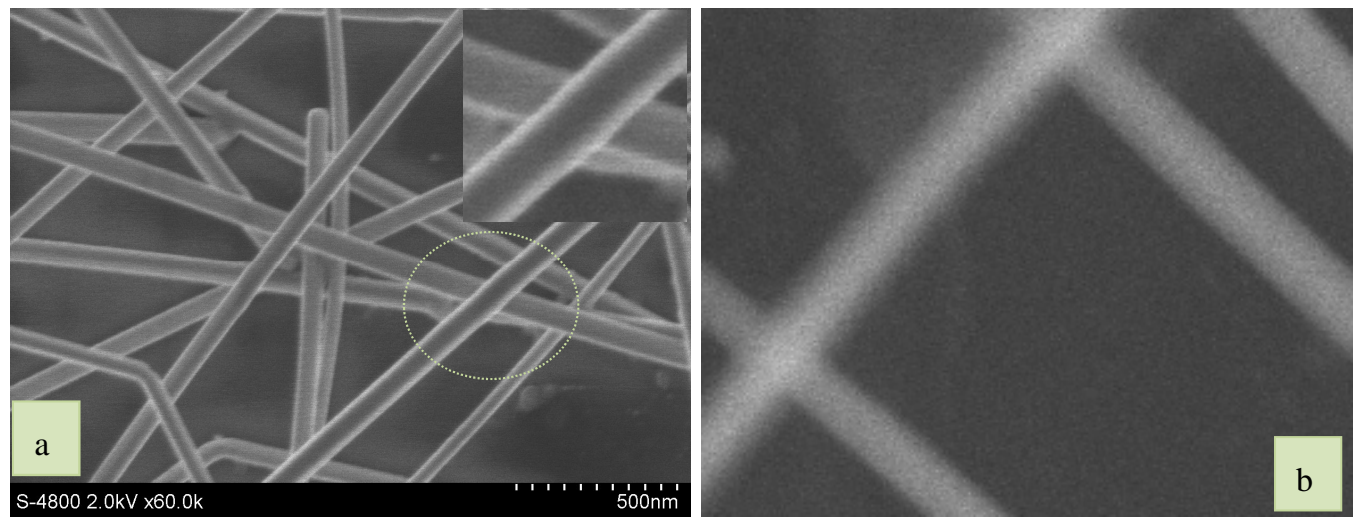

Fig. 2. AgNWs before thermal annealing (a) and AgNWs after thermal annealing (b).

Recently, to evaluate optical and electrical properties of the thin films, the concept of aspect ratio $\sigma_{D C} / \sigma_{O P}$ often used. The higher the aspect ratio is the better the properties of TCO thin film. 


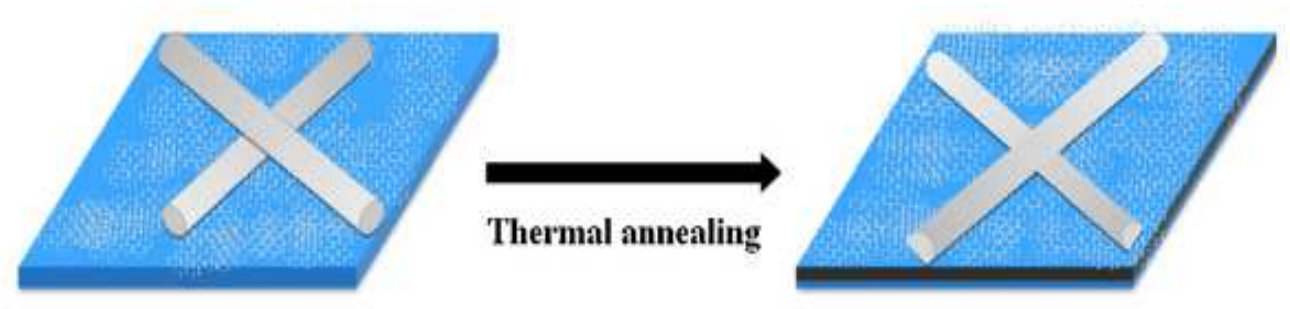

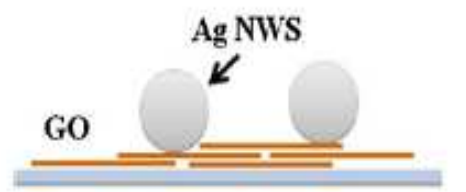

Suhstrate

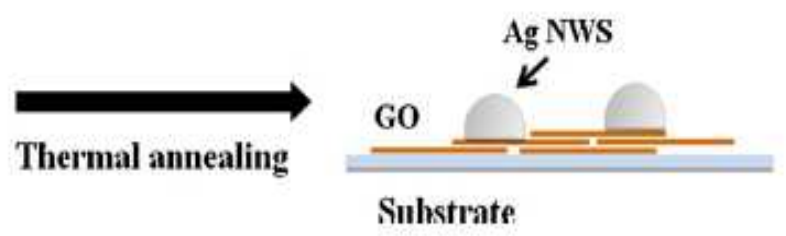

Sulhstrate

Fig. 3. Schematic image showing the effects of thermal annealing in the rGO/Ag NW hybrid film [8].

The transmittance and sheet resistance for thin films are related by (1) expression, where $\sigma_{O P}$ is the optical conductivity (here quoted at $550 \mathrm{~nm}$ ) and $\sigma_{D C}$ is the DC conductivity of the film $[9,10]$.

$$
\tau(\lambda)=\left(1+\frac{188.5}{R_{S}} \frac{\sigma_{O P}(\lambda)}{\sigma_{D C}}\right)^{-2}
$$

Follow J. N. Coleman, the minimum industry standards for these potential ITO replacements should have a sheet resistance less than $100 \Omega \mathrm{sq}^{-1}$, coupled with a light transmittance higher than $90 \%$ (at $550 \mathrm{~nm}$ ). Employing $\sigma_{D C} / \sigma_{O P}$ ratio of materials is greater than 35 [15].

Previous work has demonstrated a maximum value of $\sigma_{D C} / \sigma_{O P}=25$ for acid-treated films of single wall carbon nanotubes (SWNTs) [4] and the maximum value of $\sigma_{D C} / \sigma_{O P}=11$ for graphene films and $\mathrm{rGO}$ thin films have $\sigma_{D C} / \sigma_{O P} \approx 0.01597\left(T=80 \%\right.$ and $\left.R_{S}=10^{4}-10^{5} \Omega \mathrm{sq}^{-1}\right)[11$, 12]. These values are too low in application for optical devices. For enhancement the aspect ratio $\sigma_{D C} / \sigma_{O P}$, the combination of as-deposited graphene with other nano-structured material is effective solution. In our work, the best value of $\sigma_{D C} / \sigma_{O P}$ can be easily calculated for the hybrid film $\left(T=77 \%\right.$ and $\left.R_{S}=10.7 \Omega \mathrm{sq}^{-1}\right)$, which is $\sigma_{D C} / \sigma_{O P}=126$.

In the Table 1, the samples are prepared with the same conditions but with different annealing temperatures from $120^{\circ} \mathrm{C}$ to $180^{\circ} \mathrm{C}$ and we found that the annealing temperature at $170^{\circ} \mathrm{C}$ has the aspect ratio of the hybrid thin films is highest (Fig. 6). As above explanation, at $170^{\circ} \mathrm{C}$, the $\mathrm{AgNW}$ s contacts became joint together, which significantly decreased the resistance of network.

Unfortunately, when the annealing temperature is higher $170^{\circ} \mathrm{C}$ the transmittance of $\mathrm{Ag}$ NWs/rGO films decrease slightly (Fig. 4) although their sheet conductivities also increase (Fig. 5). Results in their aspect ratio decrease in compare to the hybrid film prepared at $170^{\circ} \mathrm{C}$. Follow $\mathrm{C}$. Erik [13], at high annealing temperature $\left(>170^{\circ} \mathrm{C}\right)$, the melting phenomenon at contact point exhibits clearly improving the contacts but also increase the scattering area at contact points. 
68 INVESTIGATION OF THERMAL ANNEALING CONDITION ON THE OPTICAL AND ELECTRICAL PROPERTIES...

Table 1. The optical and electrical properties of hybrid silver nanowires/rGO film with respect to different thermal annealing.

\begin{tabular}{cccc}
\hline \hline Temperature annealing & $\mathrm{R}(\Omega / \mathrm{sq})$ & $\mathrm{T} \%(550 \mathrm{~nm})$ & $\sigma_{D C} / \sigma_{O P}$ \\
\hline \hline $120^{\circ} \mathrm{C} 130^{\circ} \mathrm{C}$ & 3526.8 & 77.878 & 40.272653 .1731 \\
$140^{\circ} \mathrm{C}$ & 22 & 78 & 64.7745 \\
$150^{\circ} \mathrm{C}$ & 18.3 & 77.7 & 76.1922 \\
$160^{\circ} \mathrm{C}$ & 15.2 & 77.5 & 91.2373 \\
$170^{\circ} \mathrm{C}$ & 10.7 & 77 & 126.189 \\
$180^{\circ} \mathrm{C}$ & 8.2 & 71.2 & 124.182 \\
\hline \hline
\end{tabular}

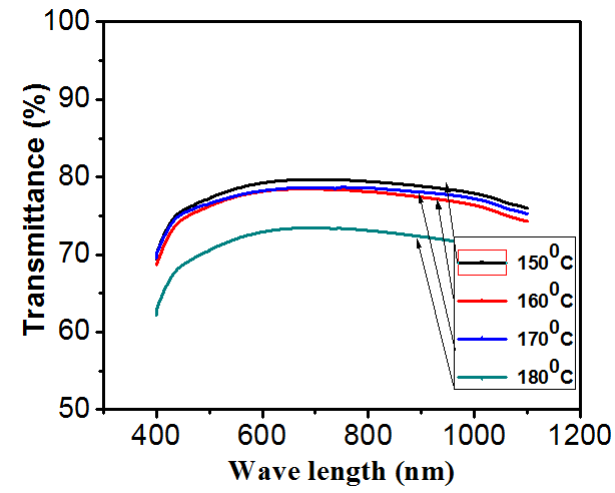

Fig. 4. Transmittance of the hybrid rGO/AgNWs electrode without including the substrate transmittance.

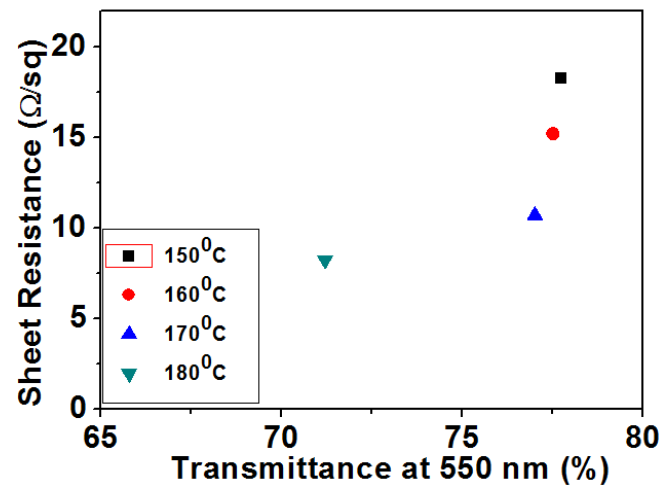

Fig. 5. Sheet resistance vs. transmittance of hybrid $\mathrm{rGO} / \mathrm{AgNW}$ s electrode with respect to different annealing temperature.

As mention above, the preparing process at low temperature can get the advantage in application of the hybrid film to the PET substrate. The initial results can get $T=70 \%$ and $R s=53 \Omega \mathrm{sq}^{-1}$, not as good as on a glass substrate (Fig. 7). However these films are stable under flexing for many cycles. To test this, we monitored the sheet resistance during bending with the $\mathrm{Ag} \mathrm{NWs} / \mathrm{rGO}$ film in tension. The films were bent from $180^{\circ} \mathrm{C}$ to $100^{\circ} \mathrm{C}$ before being relaxed. The sheet resistance of the hybrid film has no change over 400 bend cycles shown in Fig. 8.

\section{CONCLUSION}

In this work, we attempt to resolve nearly all of the current problems with hybrid AgNWs and reduced graphene oxide by employing spin coating process of rGO onto AgNWs networks at low temperatures. We report an inexpensive, fast and facile method for fabricate a flexible hybrid electrode from silver nanowires (Ag NWs) with rGO coating on arbitrary substrate. Embedding the AgNWs network in the conductive rGO matrix offers many advantages including improved wire to wire junction conductance, smooth surface morphology, excellent mechanical adhesion, flexibility. To increase adhesion between the GO and Ag NWs, and to raise the electroconductivities of the AgNWs through decomposition of the PVP and improved contact between the AgNWs 


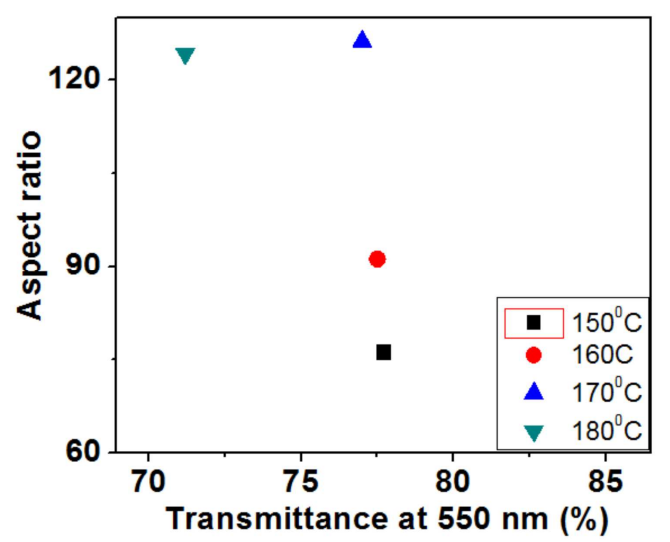

Fig. 6. The $\sigma_{D C} / \sigma_{O p}$ aspect ratio of the $\mathrm{rGO} / \mathrm{AgNW}$ sybrid with different annealing temperature.

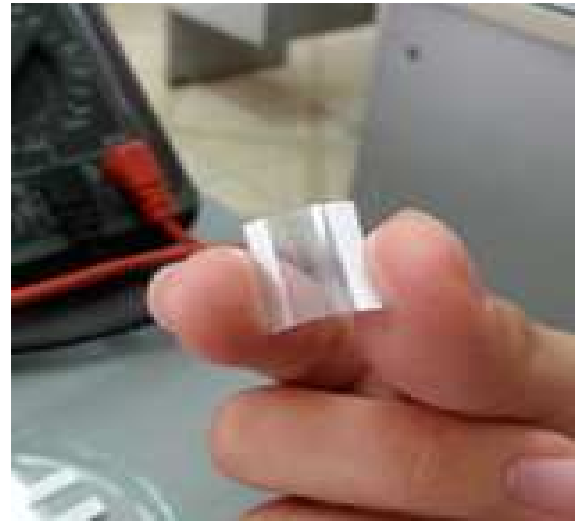

Fig. 7. Actualy image of $\mathrm{rGO} / \mathrm{AgNW}$ on PET substrate.

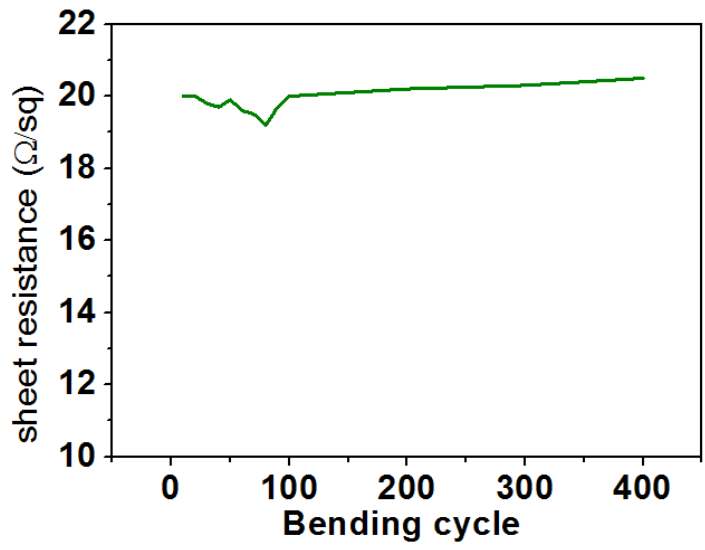

Fig. 8. Investigated resistance of $\mathrm{rGO} / \mathrm{AgNW}$ hybrid thin film after bending cycles.

and $\mathrm{GO}$, the GO/Ag NW hybrid transparent conducting films were annealed at $170^{\circ} \mathrm{C}$ for $30 \mathrm{~min}$ in an atmosphere ambient.

\section{ACKNOWLEDGMENT}

This research is funded by Vietnam National University - Ho Chi Minh City (VNU-HCM) under grant number B2012-18-12T.

\section{REFERENCES}

[1] C. H. Chung, T. B. Song, B. Bob, R. Zhu, and Y. Yang, Nano Research 5(11) (2012) 805-814

[2] H. T. Thu and T. Q. Trung, H. T. Thu and T. Q. Trung, Journal of Science and Technology 50(1B) (2012) $472-480$. 
70 INVESTIGATION OF THERMAL ANNEALING CONDITION ON THE OPTICAL AND ELECTRICAL PROPERTIES...

[3] Tran Quang Trung, Le Thi Lua, Tran Van Tam, Nguyen Thi Phuong Thanh,Huynh Tri Phong, Huynh Tran My Hoa. Journal of Science and Technology 50 (1B) (2012) 425-431.

[4] David S. Hecht, LiangbingHuandGlen Irvin, Advanced Materials 23 (2011) 1482-1513.

[5] T. Tokuno, M. Nogi, J. Jiu, and K. Suganuma, Nanoscale Research Letters 7(1) (2012) 281.

[6] Y. Ahn, Y. Jeong, an Y. Lee, ACS Appl Mater Interfaces 4 (12) (2012) 6410-4.

[7] I. N. Kholmanov, C. W. Magnuson, A. E. Aliev, R. S. Ruoff, Nano Lett. 12(11) (2012) 5679-5683.

[8] Y. S. Yun, D. H. Kim, B.Kim, H. H. Park, and H-J. Jin, Synthetic Metals 162(15-16) (2012) 1364-1368.

[9] S. De, T. M. Higgins, P. E. Lyons, E. M. Doherty, P. N. Nirmalraj, W. Blau, J. J. Boland, and J. N. Coleman, ACS Nano 3(7) (2009)1767-1774.

[10] M. Dressel and G. Gruner, Electrodynamics of Solids: OpticalProperties of Electrons in Matter; Cambridge University. Cambridge, 2002.

[11] V. C. Tung, L-M Chen, M. J. Allen, and J. K. Wassei, Nano Lett. 9(5) (2009) 1949-1955.

[12] X. L. Li, G. Y. Zhang, X. D. Bai, X. M. Sun, X. R. Wang, E. Wang, H. Dai, Nature Nanotechnology 3 (2008) $538-542$

[13] Erik C. Garnett and Mark L. Brongersma, Nature Materials 11 (2012) 241-249.

[14] L. Yang, T. Zhang, H. Zhou, S. C. Price, B. J. Wiley, and W. You, ACS Applied Materials \& Interfaces 3(10) (2011) 4075-4084.

[15] Y. Liu, Q. Chang and L. Huang, J. Mater. Chem. C 1(17) (2013) 2970-2974. 